\title{
The Origin of Ekman Flow in a Cavity Subject to Impulsive Rotational Motions
}

\author{
WEN-JEI YANG ${ }^{\mathrm{a}, *}$, HIROSHI OHUE ${ }^{\mathrm{b}}$ and GENSHI KAWASHIMA ${ }^{\mathrm{b}}$ \\ ${ }^{a}$ Department of Mechanical Engineering and Applied Mechanics, University of Michigan, Ann Arbor, \\ Michigan 48109-2125, USA; ' Department of Mechanical Engineering, Musashi Institute of Technology, \\ Tamazutsumi, Setagaya-ku, Tokyo 158-8557, Japan
}

(Received 30 March 2000; In final form 27 May 2000)

\begin{abstract}
An experimental study is performed to disclose the origin of Ekman flow on the surfaces of a rotating drum resulting from fluid-structure interaction after an impulsive start of motion (referred to as the spin-up process) or an impulsive stop (the spin-down process). Laser Doppler velocimetry (LDV) is employed to determine instantaneous distribution of both the radial and angular velocity components in the flow field inside the rotating drum. From these results, the secondary flow and the time history of the Ekman boundary layer thickness are determined. The tracer/light sheet method is also engaged to enable real-time visualization of flow patterns. Fluid viscosity, drum size and rotational speed are varied to determine their effects on fluid-structure interactions. Results may be applied to cavity flow in rotating machinery.
\end{abstract}

Keywords: Ekman flow; Cavity space; Impulsive rotational motion; LDV; Tracer/light sheet method

\section{INTRODUCTION}

The Ekman boundary layer is generated in a fluid layer near a rotating disk in which velocity vectors are twisted in a radial direction in the presence of the disk surface (Ekman, 1905). The flow inside this layer is radially outward when the disk is subjected to a sudden acceleration (a spin-up process) and is radially inward when the disk is subjected to a sudden deceleration (a spin-down process). The Ekman boundary layer is the primary factor inducing secondary flow inside enclosed vessels. Although there is an abundance of literature pertinent to the Ekman boundary layer, little effort has been made to investigate the Ekman boundary layer phenomena inside enclosed vessels.

An unsteady motion of fluid in rotating cylinders was introduced by Greenspan and Howard (1963) and Greenspan (1968). Later, Benton and

*Corresponding author. Tel.: 734 764-9910, Fax: 734747 3170, e-mail: dwakin@umich.edu 
Clark (1974) investigated flow behavior in a cylinder subjected to a spin-up process.

More recently, a series of studies were conducted on unsteady transport phenomena in rotating drums with annular space between two concentric cylinders subjected to a sudden acceleration or deceleration. These studies include measurements of velocity by means of LDV (Kawashima and Yang, 1988), hydrodynamic theory (Yang and Kawashima, 1990a), unsteady recirculating flow with inner surface heating measured by flow visualization using the tracer/light sheet method (Kawashima and Yang, 1990a), flow patterns measured by means of double-exposure photography combined with temperature measurement by means of thermocouples (Kawashima and Yang, 1990b, 1992a; Yang and Kawashima, 1990b), comparison of results obtained by the tracer/light sheet and LDV methods (Yang et al., 1992), observation of velocity fields in a plane parallel to the axis of a rotating drum (Kawashima and Yang, 1991), thermal field visualization by means of microencapsulated temperature-sensitive liquid crystal and image processing (Kawashima et al., 1992b,c), formation of Ekman boundary layers (Ohue et al., 1995), temperature distribution inside a rotating drum with inner surface heating or cooling (Ohue et al., 1996a, b, 1998) and imposition of three distinct thermal conditions; adiabatic, heating and cooling, on the inner cylinder surface (Kawashima et al., 1999). This study represents a further continuation of this series.

The LDV is employed to determine the instantaneous distribution of both the radial and angular velocity components in the flow field inside the rotating drum. From these results, the secondary flow and the time history of the Ekman boundary layer thickness are determined. The tracer/light sheet method is also engaged to enable the realtime visualization of flow patterns. The fluid viscosity, drum size and rotational speed are varied to determine their effects on fluid-structure interactions.

\section{EXPERIMENTAL APPARATUS}

The experimental apparatus used in this study is schematically illustrated in Figure 1. Two types of drum were tested whose size is shown in Figure 2. The drum consisted of an outer cylinder (inside diameter: $D_{1}=240 \mathrm{~mm}$ ), an inner cylinder (outside diameter: $D_{2}=80 \mathrm{~mm}$ ) and two circular plates for the sidewalls of $10 \mathrm{~mm}$ thickness. The widths of the drums $(B)$ were $60 \mathrm{~mm}$ (drum I) and $30 \mathrm{~mm}$ (drum II). The outer cylinder and the two sidewalls were

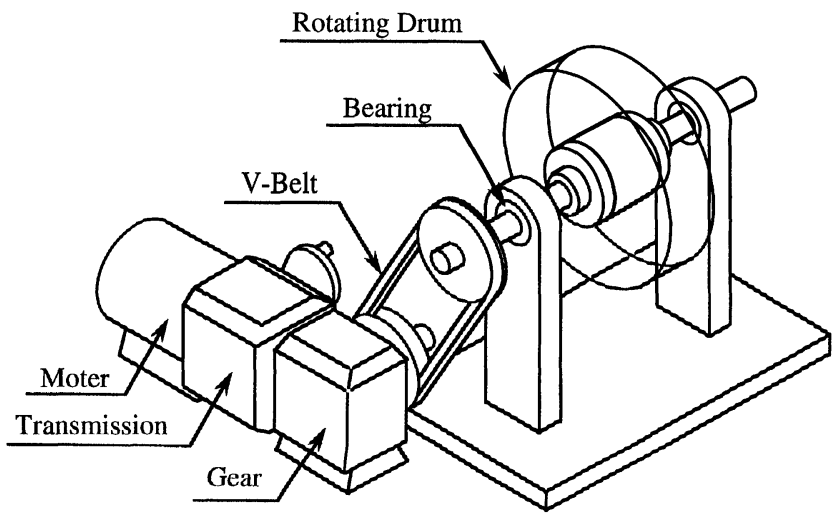

FIGURE 1 A schematic of experimental apparatus. 


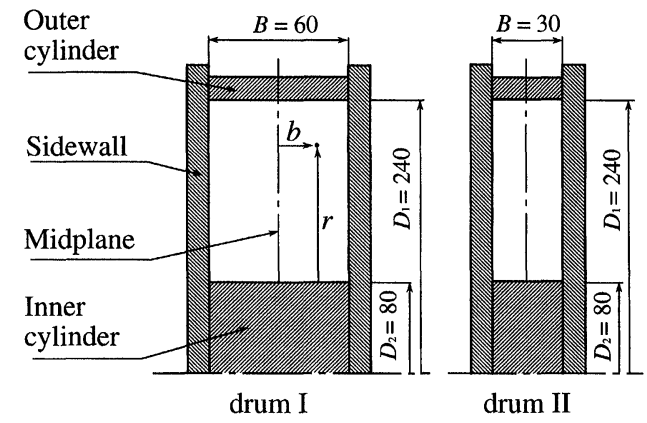

FIGURE 2 Dimensions of test drums.

made of transparent acrylic for illumination and observation. These drums were supported by bearings and driven by a variable-speed motor via a gear and a v-belt. The axis of the drum was arranged in a horizontal position. The drum was suddenly accelerated from being at rest to a steady rotational state, spin-up process, or suddenly stopped from at a steady rotational state, spindown process. It took about $0.3 \mathrm{sec}$ for the drum to achieve a steady rotational state in the spin-up case, and about $0.8 \mathrm{sec}$ to come to rest in the spindown case. These time lags are negligible for the experiments. The speeds of rotation were 0.65 and $1.26 \mathrm{rad} / \mathrm{sec}$. The drums were filled with a water or a $50 \%$ glycerin/water solution.

Figure 3 shows the illuminated plane for flow visualization. A $16 \mathrm{~mW} \mathrm{He}-\mathrm{Ne}$ laser was used as a

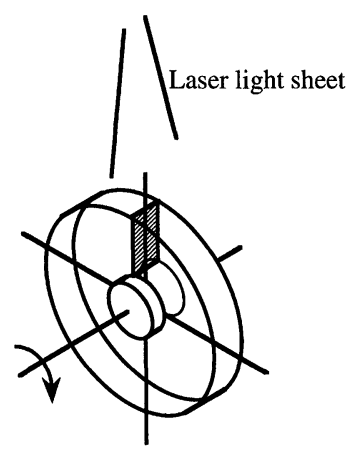

FIGURE 3 Illuminated plane for visualization. light source. The test liquids were seeded with light-scattering nylon microparticles, having a specific weight of 1.02 and a diameter of $150 \mu \mathrm{m}$, for flow visualization. The light sheet cut the meridional plane in the drum. The room temperature remained constant throughout the experiments and flow visualization experiments were conducted in a dark room. The angular and radial velocities were measured by means of LDV at strategic locations on the meridional plane.

\section{EXPERIMENTAL RESULTS}

\section{Angular Velocity}

In the interest of brevity, only some typical features of the transient flow in the drum at therotational speed of $1.26 \mathrm{rad} / \mathrm{sec}$ are presented here.

The angular velocity were measured by means of LDV at 270 (for drum I) and 135 (for drum II) strategic locations on the meridional plane. Figures 4 and 5 depict the angular velocity distributions at $10 \mathrm{sec}$ after the start of the spin-up process from at rest to $1.26 \mathrm{rad} / \mathrm{sec}$. Figure 4 is for drum I, (a) water and (b) $50 \%$ glycerin/water solution, while Figure 6 is for drum II. In order to display the distribution more clearly, the data near the sidewall $(b=-30$ and $-28 \mathrm{~mm}$ for drum I, $b=-15$ and $-14 \mathrm{~mm}$ for drum II) and inner cylinder $(r=40$ and $45 \mathrm{~mm})$ are omitted. It is seen in Figures 4 and 5 that the fluid near the outer and the inner cylinder and the sidewalls is accelerated in the spin-up process. Angular velocity is observed to drop in a region near the sidewalls. As shown later in Figure 7, the inward flow which reaches the inner cylinder turns radially outward, thus forming a vortex near the inner cylinder wall. This vortex initiates the onset of a disturbance in the angular velocity distribution. In the case of a liquid of lower viscosity, as seen in Figures 4(a) and 5(a), the disturbance is observed near the inner cylinder wall. 


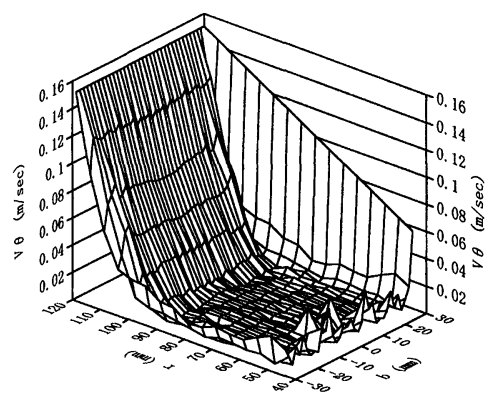

(a) water

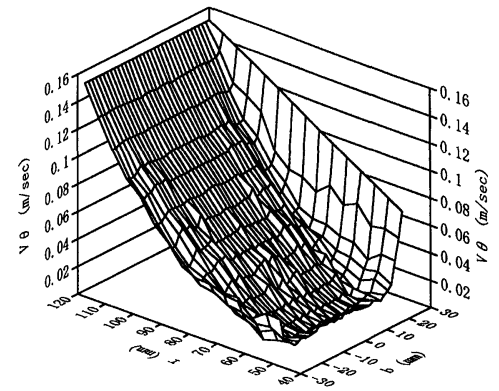

(b) $50 \%$ glycerin/water solution

FIGURE 4 Angular velocity distribution on meridional plane inside drum I under a spin-up process from a rest to $1.26 \mathrm{rad} / \mathrm{sec}$ at $10 \mathrm{sec}$ after the initiation.

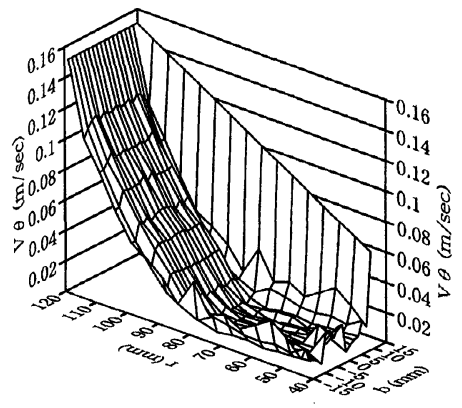

(a) water

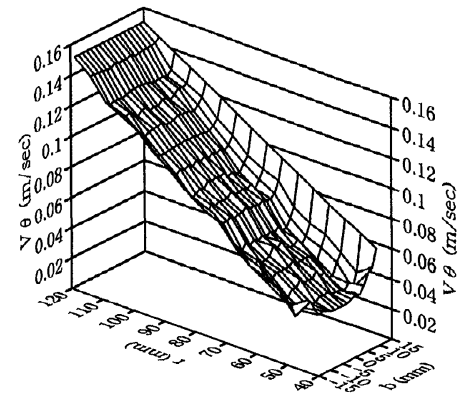

(b) $50 \%$ glycerin/water solution

FIGURE 5 Angular velocity distribution on meridional plane inside drum II under a spin-up process from a rest to $1.26 \mathrm{rad} / \mathrm{sec}$ at $10 \mathrm{sec}$ after the initiation.

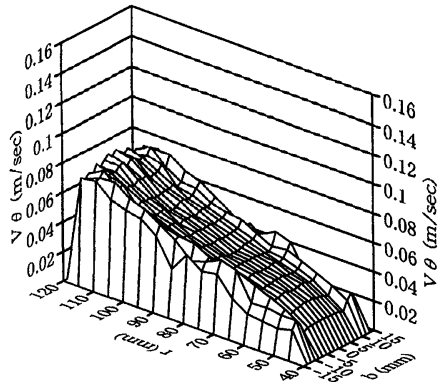

(a) water

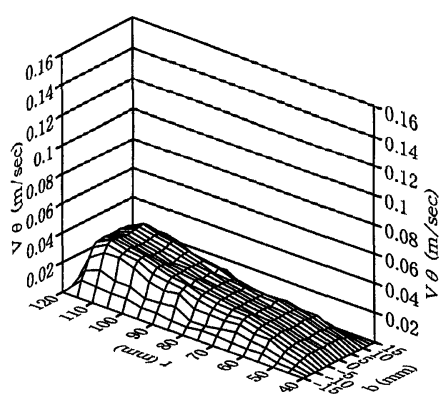

(b) $50 \%$ glycerin/water solution

FIGURE 6 Angular velocity distribution on meridional plane inside drum II under a spin-down process from $1.26 \mathrm{rad} / \mathrm{sec}$ to a rest at $10 \mathrm{sec}$ after the initiation. 


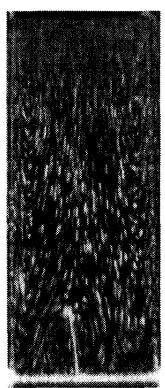

(a) Flow pattern

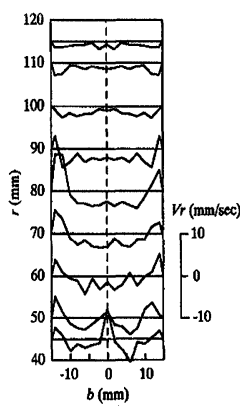

(b) Radial velocity distribution
FIGURE 7 Flow pattern and radial velocity distribution on meridional plane for water inside drum II under a spin-up process from a rest to $1.26 \mathrm{rad} / \mathrm{sec}$ at $10 \mathrm{sec}$ after the initiation.

Figure 6 shows the angular velocity distributions after $10 \mathrm{sec}$ in the spin-down process. One observes in Figure 6 that the angular velocity distribution in the axial direction has a higher value near the sidewalls, which is an effect of secondary flow. The disturbance in the angular velocity distribution for water is larger than that for the $50 \%$ glycerin/water solution.

\section{Secondary Flow}

The occurrence of secondary flow in a rotating drum is observed during a spin-up or spin-down process. Figures 7 and 8 depict the flow pattern and their respective radial velocity distributions on

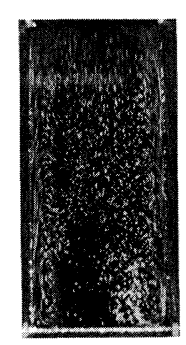

(a) Flow pattern

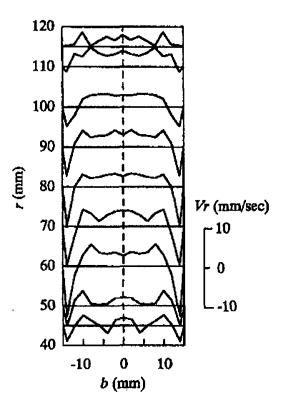

(b) Radial velocity distribution
FIGURE 8 Flow pattern and radial velocity distribution on meridional plane for water inside drum II under a spin-down process from $1.26 \mathrm{rad} / \mathrm{sec}$ to a rest at $10 \mathrm{sec}$ after the initiation. the meridional plane for water in the drum II after $10 \mathrm{sec}$ in the spin-up and spin-down process. Radial velocities were measured by LDV at 72 strategic locations on the meridional plane in drum II.

As shown in Figure 7, the fluid near the sidewall flows radially outward during the spin-up process, thus developing the Ekman boundary layer. Meanwhile, the fluid at the midplane is forced toward the inner cylinder side. Consequently, a secondary flow is generated symmetrically in the meridional plane. The radially inward flow interacts with the inner cylinder wall to generate a vortex, as seen in the flow pattern, thus causing the disturbance in the angular velocity distribution near the inner cylinder.

In the spin-down process as shown in Figure 8, the process of inducing a recirculating flow is opposite to that of the spin-up process. The Ekman boundary layer developed over the sidewall has radially inward flow. Since the Ekman boundary layer has its exit port near the inner cylinder wall, the fluid there is forced to exit in the axial direction, and then turns radially outward in order to attain flow symmetry with respect to the midplane.

\section{The Ekman Boundary Layer}

Figures 9 and 10 show the time history of the Ekman boundary layer thickness for the spin-up and spin-down processes. The Ekman boundary layer develops immediately after the initiation of the spin-up or spin-down processes. The thickness gradually decreases with time from about 6 sec late in the spin-up process, as shown in Figure 9. In contrast, the thickness gradually increases with time until it comes to rest in the spin-down process, in Figure 10. When compared relative to differences in the fluid viscosity, drum size and rotational speed, the Ekman boundary layer becomes thicker for higher viscosity $(50 \%$ glycerin/water solution), for the wider width of drum (drum I) and for the lower rotational speed $(0.65 \mathrm{rad} / \mathrm{sec})$ in both the spin-up and spin-down processes. 


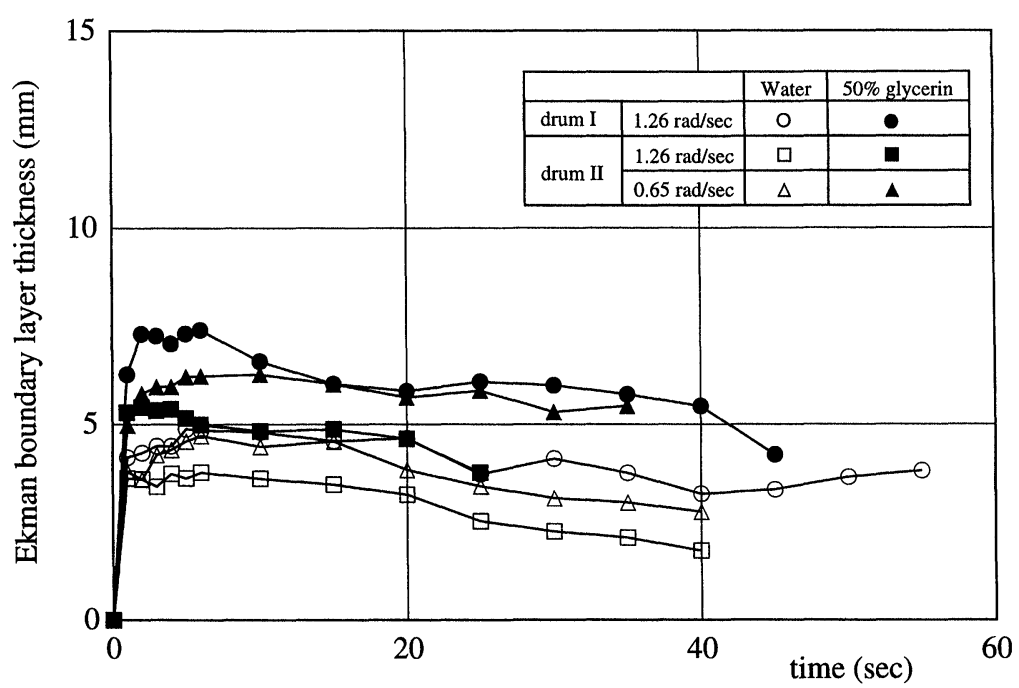

FIGURE 9 Time history of Ekman boundary layer thickness under a spin-up process.

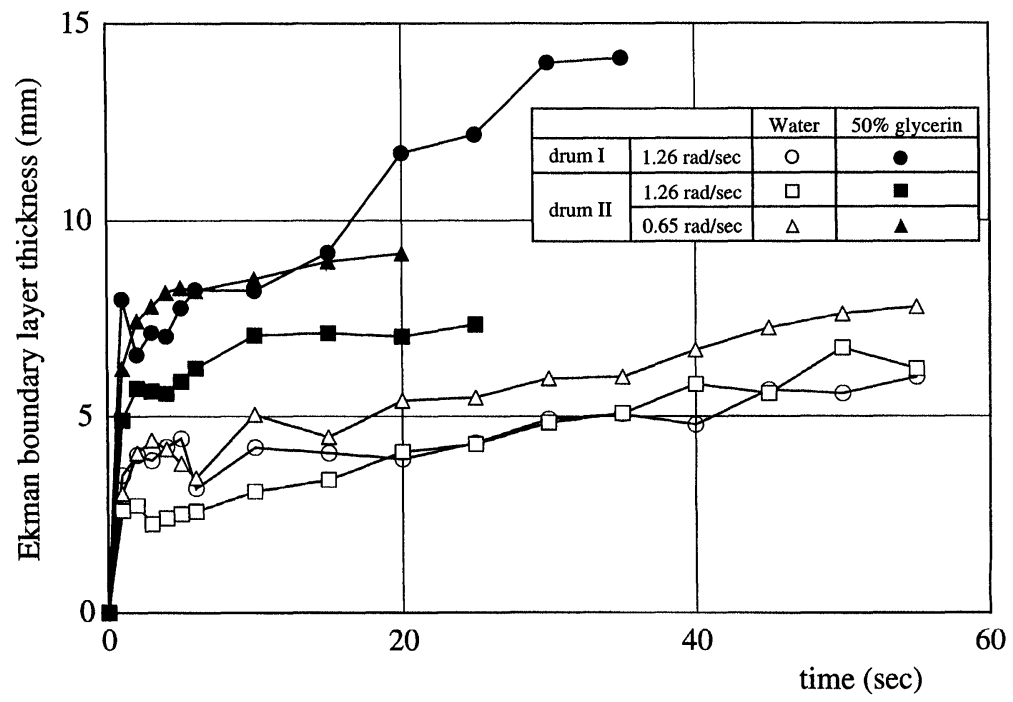

FIGURE 10 Time history of Ekman boundary layer thickness under a spin-down process.

\section{CONCLUSIONS}

The feature of flow-structure inside a drum under the spin-up and spin-down processes have been investigated by means of LDV measurements and flow visualization. The following conclusions are derived from the results of these experiments:

(1) The formation of Ekman boundary layers on both sidewalls results in a pair of recirculating flows, as seen in flow patterns and as also evidenced in radial velocity distributions.

(2) The recirculating flow causes a vortex near the inner cylinder wall. 
(3) This vortex initiates the onset of a disturbance in the angular velocity distribution.

(4) The Ekman boundary layer which developed immediately after the start of the spin-up process gradually decreases with time, while it gradually increases during the spin-down process.

(5) The thickness of the Ekman boundary layer increases with fluid viscosity, drum width and rotational speed.

\section{NOMENCLATURE}

\section{$B \quad$ drum width}

$b$ axial distance from midplane of the drum

$D_{1}$ inside diameter

$D_{2}$ outside diameter

$r$ radial distance from the center of the drum

$V_{\theta}$ angular velocity

$V_{r}$ radial velocity

\section{References}

Benton, E. P. and Clark, A. (1974) "Spin-up", Annual Review of Fluid Mechanics, 6, 257-280, Annual Reviews Inc. California.

Ekman, V. W. (1905) On the Influence of the Earth's Rotation on Ocean Currents, Ark. Mat. Astron. Fys., 2.

Greenspan, H. P. and Howard, L. N. (1963) On a Time Dependent Motion of Rotating Fluid, Journal of Fluid Mechanics, 17, 385-404.

Greenspan, H. P. (1968) The Theory of Rotating Fluids, Cambridge University Press, Cambridge.

Kawashima, G. and Yang, W. J. (1988) Unsteady Flow in Rotating Drums using Laser Doppler Velocimetry, Experiments in Fluids, 6(3), 165-171.

Kawashima, G. and Yang, W. J. (1990a) Light-sheet Visualization of Unsteady Recirculating Flow Inside Rotating Drums, Flow Visualization V, Hemisphere, pp. 633-639.
Kawashima, G. and Yang, W. J. (1990b) Experimental Study on Unsteady Recirculating Flow in Rotating and Stationary Drum with Inner Surface Heating, $A S M E F E D, 92$, $219-224$.

Kawashima, G. and Yang, W. J. (1991) Determination of Unsteady Flow inside Rotating Drums by Flow Visualization with Double-Exposure Photographs, ASME FED, 106, $41-46$.

Kawashima, G. and Yang, W. J. (1992a) Unsteady Recirculating Flow in a Steady Rotating Drum due to Inner Surface Heating, ASME FED, 133, 273-274.

Kawashima, G., Yang, W. J. and Ohue, H. (1992b) Image Processing of Flow Patterns in Rotating Drums with Inner Surface Heating, International Seminar on Imaging in Transport Processes (Athens), pp. 197-205.

Kawashima, G., Yang, W. J. and Ohue, H. (1992c) Visualization of Unsteady Flow in Rotating Drums, Flow Visualization VI, Springer Verlag, pp. 62-66.

Kawashima, G., Ohue, H. and Yang, W. J. (1999) Visualization of Unsteady Flow inside Rotating Drums, Proceedings of 2nd Pacific Symposium on Flow Visualization and Image Processing (Hawaii), PF051.

Ohue, H., Yang, W. J. and Kawashima, G. (1995) Ekman Boundary Layers and Energy Dissipation in Rotating Drums During Spin-down Process, International Journal of Rotating Machinery, 2(2), 113-121.

Ohue, H., Yang, W. J. and Kawashima, G. (1996a) Transient Convective Heat Transfer in a Concentric Drum with Inner Surface Cooling, Proceedings of 9th International Symposium on Transport Phenomena in Thermal-Fluids Engineering (Singapore), II, 945-950.

Ohue, H., Yang, W. J. and Kawashima, G. (1996b) Unsteady Convection in a Rotating drum with Inner Surface Heating, Proceedings of 6th International Symposium on Transport Phenomena and Dynamics of Rotating Machinery (Hawaii), II, $431-439$.

Ohue, H., Yang, W. J. and Kawashima, G. (1998) Unsteady Convective Heat Transfer in a Rotating Concentric Drum with Inner Surface Cooling, Proceedings of 7 th International Symposium on Transport Phenomena and Dynamics of Rotating Machinery (Hawaii), C, 1721-1729.

Yang, W. J. and Kawashima, G. (1990a) Hydrodynamics in Unsteady Rotating Drums, Transport Phenomena in Rotating Machinery, Hemisphere, pp. 51-61.

Yang, W. J. and Kawashima, G. (1990b) Experimental Study on Unsteady Convection in Rotating Drums with Inner Surface Heating, Preprints of 3rd International Symposium on Transport Phenomena and Dynamics of Rotating Machinery (Hawaii), 1, 156-166.

Yang, W. J., Ohue, H. and Kawashima, G. (1992) Experimental Study by LDV and Flow Visualization on the Unsteady Flow Pattern in Rotating Drums, Proceedings of 4th International Symposium on Transport Phenomena and Dynamics of Rotating Machinery (Hawaii), B, 555-561. 

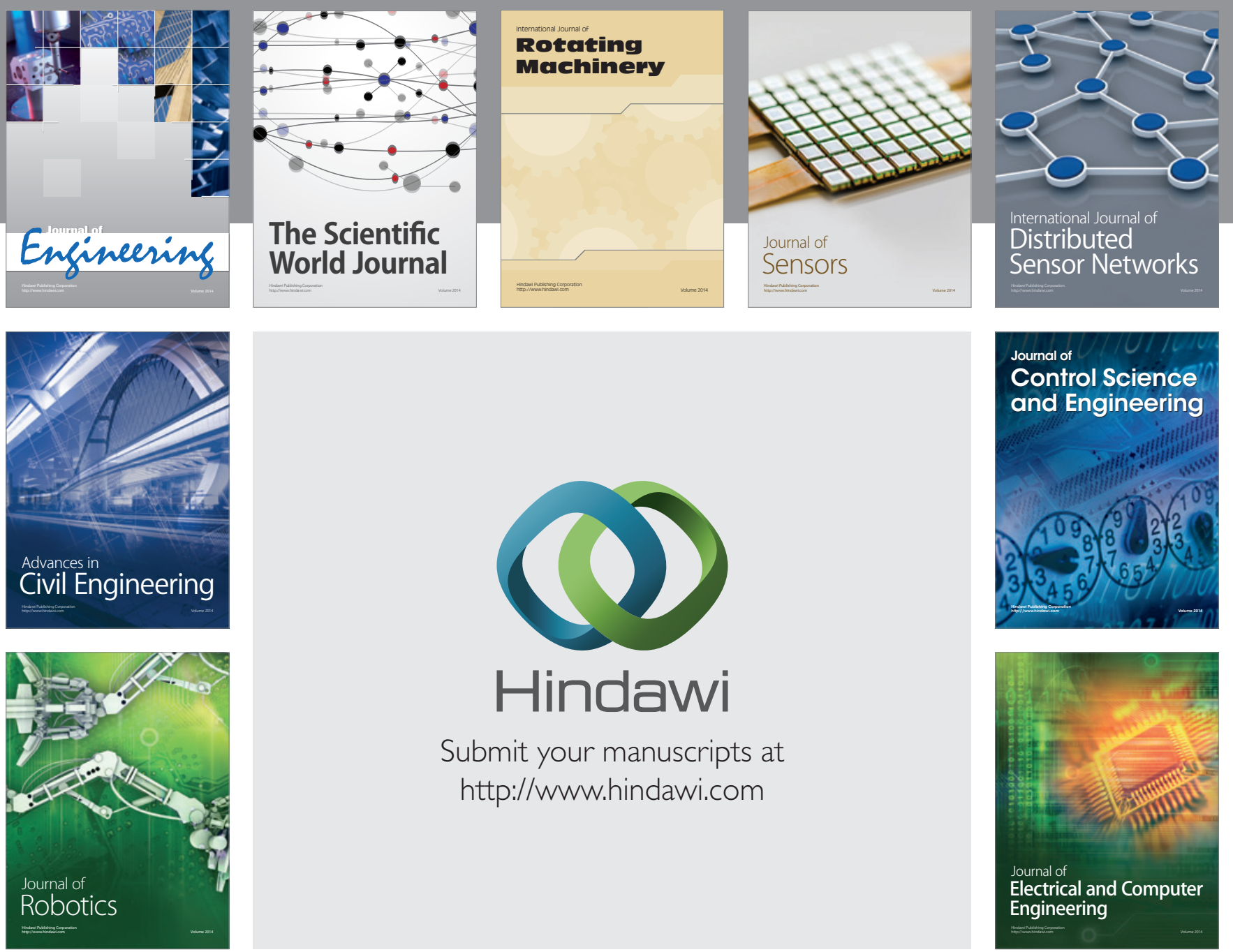

Submit your manuscripts at

http://www.hindawi.com
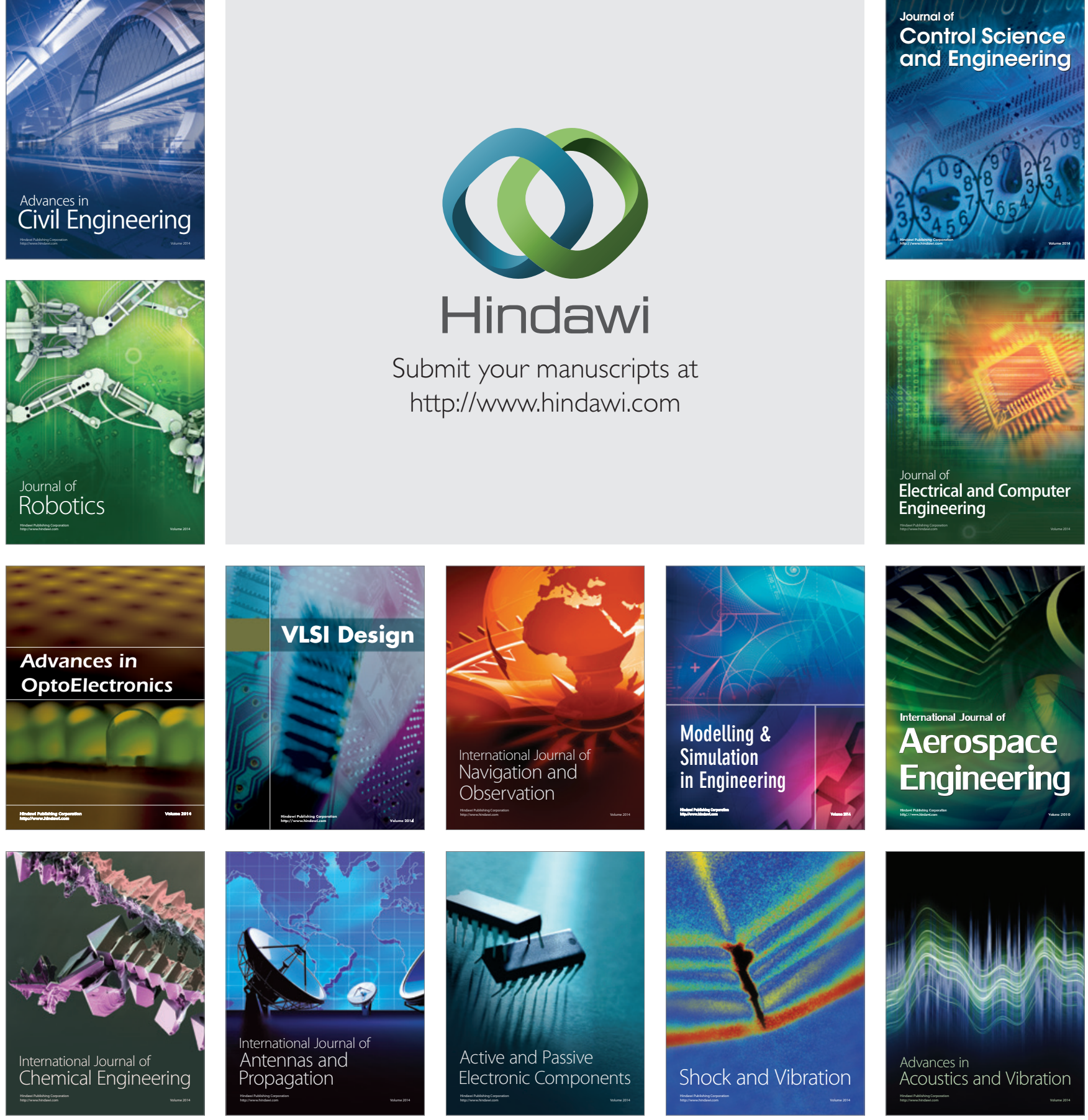